\title{
EDITORIAL
}

\section{The Edinburgh heart valve study}

\section{K M Taylor}

The durability of artificial heart valves is a key consideration, particular in an increasingly elderly population. So which type of artificial valve is preferable-mechanical or bioprosthetic?
$\mathrm{D}$ urability has always been a crucial performance indicator for artificial heart valves. There is the intrinsic durability of the valve itself, as constructed by the manufacturer, but it is the durability of the implanted valve in the individual patient which is the real issue, particularly, but not solely, for the patient. Progress in the design and manufacture of artificial heart valves has arisen from information gained from pulse duplicator studies, from fatigue testing, and from animal implants-but the bottom line is durability once implanted in the patient, and the longer the follow up the better.

The Edinburgh heart valve study of 533 patients, who had their valve implant(s) between 1975 and 1979, now reports comparative clinical outcome for mechanical versus bioprosthetic valves at 20 years. ${ }^{1}$ The present report supplements a 12 year follow up, published in 1991. ${ }^{2}$ The original study was prospective and randomised. The study design was modified in January 1977 in those patients randomised to receive a porcine bioprosthesis. Initially, the porcine valve used was the Hancock prosthesis, but, after January 1977, the Carpentier-Edwards valve was used because of its "substantial cost advantage".

The authors are to be congratulated over the length and completeness of follow up. Six patients were lost to follow up and the remaining survivors were followed up beyond 1 January 1988, giving a mean follow up of 20.4 years. The patient population in the study is younger than current patient demographics, with a mean age at operation of 54 years. Data from the UK Heart Valve Registry (UKHVR), which commenced data collection across the UK in 1986, reported a mean age at operation of 59 years in 1986, ${ }^{3}$ with progressive increase to a mean of 66 years for first time valve replacement patients in $2000 .^{4}$ In 2000 , approximately $40 \%$ of first time valve replacement patients were 70 years old or older. ${ }^{4}$

The question of the change in bioprosthetic valve of choice midway through patient recruitment must be addressed. The authors indicate

\section{ANTICOAGULATION}

The incidence of anticoagulation treatment is, as expected, different for the mechanical valve patients compared to those receiving a bioprosthesis, and also differed for patients having an aortic valve replacement (AVR) compared with those having a mitral valve replacement (MVR). Exposure to warfarin increased with the passage of time in the porcine group, rising to $33 \%$ of AVRs and $57 \%$ of MVRs at 15 years postimplant.

What then of the results? In their 12 year analysis, ${ }^{2}$ survival appeared to be better in patients receiving the mechanical valve, although the difference did not reach significance. What was evident at 12 years was the increased risk of haemorrhagic morbidity with the mechanical valve, related to the requirement for anticoagulant treatment. ${ }^{2}$

The current paper extends the mean follow up to 20 years-surely a meaningful extension of life. No significant difference was found in survival between mechanical and bioprosthetic valve implant patients, both groups having around $22-25 \%$ survival. Adding reoperation to a composite outcome measure favoured the mechanical valve (as one might expect), the difference being apparent earlier with MVR (8-10 years) compared with AVR (12-14 years).

The obligatory use of anticoagulant treatment in patients implanted with mechanical valves was again, as might be expected, associated with an increased risk of haemorrhagic complications in the mechanical valve group ( $41 \% v 28 \%)$. There was no difference in the occurrence of thrombosis or, significantly, in infective endocarditis.

\section{REOPERATION}

What is clear is the increased requirement for reoperation in patients implanted with bioprostheses. Overall, $21 \%$ of the patients required reoperation during the follow up period, but around four times more reoperations were performed in the bioprosthetic group compared to the mechanical group. The present study rightly draws attention to the not inconsiderable risks of reoperation ( $15 \%$ at 30 days and $22 \%$ at one year following operation). These data are consistent with the mortality rates following reoperation reported in the UKHVR. ${ }^{4}$ Although much lower mortality rates for reoperative valve surgery have been reported, ${ }^{56}$ overall experience with reoperative valve replacement still indicates that this is a relatively high risk procedure, and should not be undertaken lightly.

Abbreviations: AVR, aortic valve replacement; MVR mitral valve replacement; UKHVR, UK Heart Valve Registry \footnotetext{
that analyses comparing the two porcine valve types produced "very similar" results. Although no data are provided in this manuscript relating to this issue, the assumption of the relative equivalence of these two porcine bioprosthetic valves is probably acceptable for the purposes of this study.

B Block, Hammersmith Hospital, Du Cane Road, London W12 ONN, UK: scarroll@imperial.ac.uk

..................

Correspondence to: Professor KM Taylor,
Cardiothoracic Surgica
} 


\section{HAEMORRHAGE}

It has always been accepted that haemorrhagic complications will inevitably be higher in patients who require anticoagulation treatment. The patient who is in sinus rhythm, and implanted with a bioprosthetic valve, may safely and protectively be taken off warfarin anticoagulation after 6-12 weeks post-valve implant. The data from the current study would immediately confirm this perception-particularly at 10 years postimplant ( $15 \%$ mechanical $v 7.5 \%$ bioprosthetic for all bleeding episodes: $12 \%$ mechanical $v 5 \%$ bioprosthetic for all major bleeding episodes). The situation changes by 20 years, however, with a relative increase in the bioprosthetic group incidence of all major bleeding episodes. One important factor to be taken into account, however, is the progressive increase in anticoagulant treatment in the bioprosthetic valve patient group with the passage of time-at five years postoperatively, $15 \%$ of aortic and $36 \%$ of mitral bioprosthetic implantees were being treated with warfarin. By 15 years, the numbers had doubled.

\section{SUMMARY}

What do the authors of this commendable study conclude?

They recommend:

(1) mechanical valve for single MVR or combined AVR and MVR. Their argument rests on the near inevitability of anticoagulation and the "superior durability of these prostheses".

(2) mechanical valve for single AVR in patients expected to live more than 10 years (not defined), unless anticoagulation is contraindicated.

These recommendations appear valid in the context of the current study's population and time context. Inevitably, however, the question has to be asked: Is the valve replacement patient population in 2002 the same or different to that described in 1975-79?

Clearly, the patient population is different-it is significantly older, less rheumatic and more degenerative, with more co-morbidity. More AVRs are performed than MVRs, and more patients, consequently, present in sinus rhythm rather than in atrial fibrillation.

The present study, based on relatively young patients, with equivalent MVR compared to AVR, differs from the current pattern of valve replacement surgery. Their conclusions for the mid to late 70s patient population are appropriate, but less relevant to today's valve surgery practice-increasingly elderly patients, with a predominance of AVR over MVR, in sinus rhythm with the potential to avoid anticoagulants.

Currently, we have to think about the appropriate artificial valve/valve repair procedure in an increasingly elderly population. If the decision is replacement rather than repair, the latest results from the UKHVR, ${ }^{4}$ prospective since 1986, for patients having mechanical valve replacements $(n=46810)$ compared to bioprosthetic implants $(\mathrm{n}=25$ 977) report survival at 15 years postoperation for mechanical valve patients of $47.4 \%$ (95\% CI $46.3 \%$ to $48.6 \%$ ) compared to bioprosthetic valve patients of $30.3 \%$ (95\% CI $29.0 \%$ to $31.6 \%$ ). This difference is at least partially explained by patient age at the time of implant. For those patients aged 70 years and over, the survival rates at 15 years postimplant are virtually identical (mechanical $20.2 \%, 95 \%$ CI $16.5 \%$ to $24.1 \%, v$ bioprosthetic $16.6 \%, 95 \%$ CI $14.3 \%$ to $19.0 \%$ ). This relative equivalence of general durability would suggest that the pendulum of preference for mechanical valves, rightly proposed on the basis of the Edinburgh valve study findings, may be swinging back to a neutral position. If the patient demographics continue to move towards the elderly, and sinus rhythm and aortic valve replacement assumes the preponderance over atrial fibrillation, mitral valve disease, and the need for anticoagulation, the pendulum may swing even further.

\section{REFERENCES}

1 Oxenham H, Bloomfield P, Wheatley DJ, et al. Twenty year comparison of a Bjork-Shiley mechanical heart valve with porcine bioprostheses. Heart 2003;89:715-21

2 Bloomfield P, Wheatley DJ, Prescott RJ, et al. Twelve year comparison of a Biork-Shiley mechanical heart valve with porcine bioprostheses. N Engl I Med 1991·324:573-9.

3 United Kingdom Heart Valve Registry. The United Kingdom heart valve registry report 1986. London: United Kingdom Heart Valve Registry, Royal Postgraduate Medical School, Hammersmith Hospital, 1988.

4 United Kingdom Heart Valve Registry. The United Kingdom heart valve registry report 2000. London: United Kingdom Heart Valve Registry, Imperial College School of Medicine, 2002.

5 Piehler JM, Blackstone EH, Bailey KR, et al. Reoperation on prosthetic heart valves. J Thorac Cardiovasc Surg 1995; 109:30-48.

6 Akins CW, Buckley DM, Daggett WM, et al. Risk of reoperative valve replacement for failed mitral and aortic bioprostheses. Ann Thorac Surg 1998;65:1545-51

\section{ELECTRONIC PAGES}

\section{eHEART: www.heartinl.com}

The following electronic only article is published in conjunction with this issue of Heart.

Persisting fever in a patient with brucella endocarditis: occult splenic abscess

\section{B Yilmaz, H L Kisacik, S Korkmaz}

Brucella endocarditis, despite its high mortality rate with combined medical and surgical treatment, has a low occurrence rate in cases of brucellosis and has been endemic in regions surrounding Turkey. Rarely, patients with infective endocarditis with common microorganisms develop a splenic abscess. A patient is reported on with brucella endocarditis and persistent fever. An occult splenic abscess was found. This is the second reported case in the literature of brucella endocarditis with splenic abscess.

(Heart 2003;89:e20) www.heartjnl.com/cgi/content/full/89/ 7/e20 